\title{
Decision on Transfer Policy for A Car Rental Company in Multi-City Network
}

\author{
F. Tao \\ Department of Management Science and Engineering \\ School of Business \\ East China University of Science and Technology \\ China
}

\begin{abstract}
In this paper, we propose dynamic programming model for a car rental company in multi-city network to determine the transfer policy in daily operation. In the model, each city faces two types of demand, namely, inter-city and local city demands, where the former refers to a customer pick up a vehicle in one city but return it in another city and the latter refers to a customer rent a vehicle in one city and return it to the original city. Because of inter-city demand, the vehicles in each city may become unbalanced. It is necessary to transfer vehicles among these cities to satisfy the corresponding demand. Though the complexity of this problem, we analyse the transfer strategy and propose an algorithm to achieve the transfer policy.
\end{abstract}

Keywords-car rental; dynamic programming; multi-city network; transfer

\section{INTRODUCTION}

With the development of economics and the constraint on carbon emission issue, public transportation has been paid more attention on, meanwhile car rental business have the potential for growth. Therefore, many companies open branches in several cities and provide car rental service.

The analysis presented in this paper is motivated by a small rental-car company that serves in two cities in China. In our paper, the system owns a multi-city network that operations car rental business among several cities. In each city, there exists two types of demand, namely, inter-city demand (type-1) which refers to the customer rent a car from a city but return it in another city, and local-city demand (type-2) which means the customer rent a car from a city but return it to the original city (see Figure 1). Because the inter-city rental rate is much higher than the local rental rate, it is the corporate policy that a higher priority is given to the inter-city demand. Therefore, the number of cars in these two cities may become unbalanced when the inter-city demand surges in one city and remains flat in the other. As such, the company often needs to decide whether to transfer any vehicles from one city to another one at the end of daily operations. Therefore, the manager needs to decision whether to transfer cars among these cities to make sure that the demand can be satisfied and the optimal profit is achieved. Here, the fleet size of the system is predetermined parameter; however it can be released in future research.
This paper is an extension of $\mathrm{Li}$ and Tao [1], in which they only considered a two-city problem with two types of demand and investigated the optimal fleet size and vehicle transfer policy. Oliveira et al. [2] considered a vehicle-reservation assignment framework as a network-flow model and propose a relax-and-fix-based heuristic algorithm to solve the problem. You and Hsieh [3] applied constrained mixed-integer programming model to address the fleet size and transfer problem for the car-rental companies with multiple branches. Lin et al. [4] formulated a Markov decision model to study the vehicle allocation problem in automated material handling system. Goldbarg et al. [5] proposed meta-heuristic approaches to deal with traveling car rental problem. Awasthi et al. [6] presented a centralized fleet management system for cybernetic vehicles.

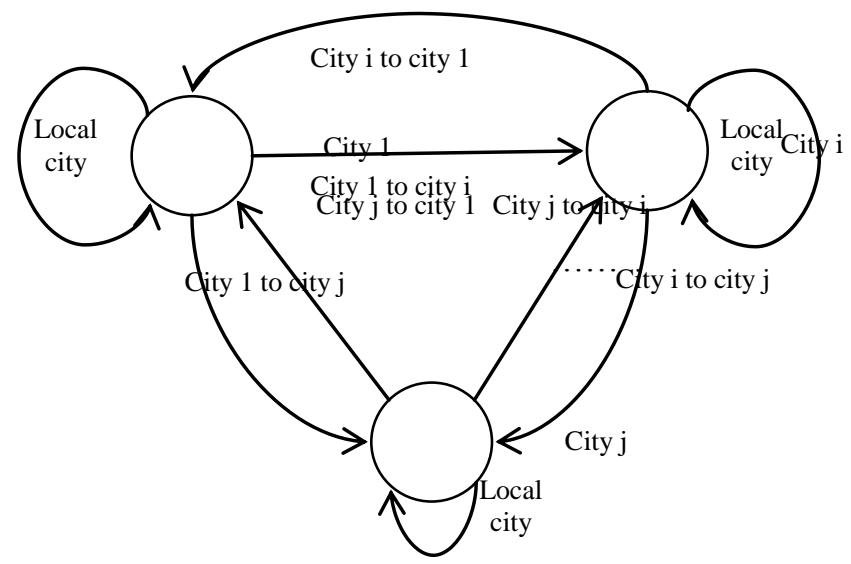

FIGURE I: MULTI-CITY NETWORK.

\section{MODEL}

Specifically, we consider a rental-car company that opens several branches serving a number of cities. Before presenting the model, define the following notations in alphabetical order.

$D_{i j}$ is the demand from city $i$ to city $j$ at city $i$, where $i, j=1,2, \ldots, l$. We assume that there are $l$ cities in the system. When $i=j$, it refers to the local round demand; otherwise if $i \neq j$, it refers to the inter-city demand. $D_{i j}$ is assumed to be dependent on $i, j$, but for any given $(i, j)$, it 
follows independent and identical distribution in each decision epoch and $E\left(D_{i j}\right)<\infty$;

$h$ is unit holding cost irrespective the car is rent or not;

$k$ is the unit transfer cost among these cities. We assume that there is no difference among all cities.

$r_{i}$ is the rental rate for type- $i$ demand, where $i=1,2$. We assume that the corresponding rental rates are the same among these cities.

$t$ is the decision epoch, we count the time index backward;

$x_{i}$ is the initial number of cars in city $i$ before transfer decision is made, $i=1,2, \ldots, l$.

$y_{i}$ is the number of cars available in city $i$ after transfer cars arriving the terminated city, $i=1,2, \ldots, l$.

$V_{t}^{n}\left(x_{1}, x_{2}, \ldots, x_{l}\right)$ is the maximum expected profit attainable when the initial number of cars in city $i$ is $x_{i}$ in decision epoch $t$, while the fleet size is $n$ which indicates that $x_{1}+x_{2}+\ldots+x_{l}=n$;

We count the time index backward, i.e., $t=0$ stands for the end of the planning horizon and period $T$ represents the beginning of the planning horizon. The sequence of the events is the following:

At the end of the planning horizon, namely, in the evening of the initial epoch, the firm decides the fleet size $n$ (e.g., total number of cars) and allocates $x_{i}$ cars to city $i$. Demand arrives periodically starting from day $T-1$.

In the morning of a typical day ${ }^{t}(\leq T-1)$, transferred cars, if any, arrive. Now, there are ${ }^{y_{i}}$ cars in city $i$.

Demand for day $t$ arrives. We assume the type-1 demand has high priority than type-2 demand as it earns more profits (rental rate for type-1 is higher than type-2). Therefore, the available vehicles will be used to satisfy the type-1 demand first, then type-2 demand, consecutively. The demand is satisfied instantly and the excessive demand is lost.

In the evening, cars are returned. The available cars in city $i$ is $x_{i}$. Now, the company needs to decide whether to transfer some vehicles from one city to another city overnight.

Repeat events 2, 3, and 4 until the end of the planning horizon.

Without loss of generality, suppose that in the evening of period $t+1$, there are $x_{i}$ cares in city $i$ and $x_{1}+x_{2}+\ldots+x_{l}=n$. In the morning of period $t$, there are $y_{i}$ cars available in city $i$ after transfer activity and $x_{1}+x_{2}+\ldots+x_{l}=n$. For simplicity, we assume that both types of customers use the car for one day only. That is, type-1 customer in city $i$ will pick up the car in the morning and return it to city $j(\neq i)$ in the evening; whereas type-2 customer in city $i$ will pick up the car in the morning and return it to city $i$ in the evening. Thus, the optimal equation can be

$$
\begin{aligned}
& V_{t}^{n}\left(x_{1}, x_{2}, \ldots, x_{l-1}, x_{l}\right)= \\
& \sum_{i=1}^{l} \max _{i=n, \sum_{i=1}^{l} y_{i}=n}\left\{\begin{array}{l}
-k \sum_{i=1}^{l}\left|y_{i}-x_{i}\right|+r_{1} \sum_{i=1}^{l} \sum_{j=1}^{l} E \min \left(y_{i}, \sum_{j=1, j \neq i}^{l} D_{i j}\right) \\
+r_{2} \sum_{i=1}^{l} E \min \left[\left(y_{i}-\sum_{j=1, j \neq i}^{l} D_{i j}\right)^{+}, D_{i i}\right]-h n \\
+E V_{t-1}^{n}\left(y_{1}+\sum_{i=2}^{l}\left(D_{i 1}-D_{l i}\right), \ldots, y_{l}+\sum_{i=1}^{l-1}\left(D_{i l}-D_{l i}\right)\right)
\end{array}\right\}
\end{aligned}
$$

The first term is the transfer cost; the second and third terms are revenue of inter-city and local-city demand, respectively; holding cost is shown by the fourth term. The last term is the expected future profit.

\section{TRANSFER POLICY}

In terms of the transfer policy, as the complexity in multicity network, it is intractable to characterize the policy as $\mathrm{Li}$ and Tao (2010). However, we can get the following conclusion.

Let a straight line with an arrow represent the transfer activity starting from the outbound city with excessive car(s) and ending with the inbound city that is lack of car(s). Therefore, we can derive the following strategy:

Lemma 1 . For any given three cities, the transfer activities among these cities cannot be a ring.

Proof. Suppose 1, 2, 3 stand for these three cities respectively. Two cases need to be concerned:

Case 1): cars are transferred from city 1 to both city 2 and 3 , in addition, some vehicles are moved between city 2 and city 3 . Without loss of generality, assume transfer $a_{1}, a_{2}$ cars from city 1 to city 2 and 3 , respectively; and move ${ }^{a_{3}}$ cars from city 2 to city 3 . Thus, the total transfer cost should be $k\left(a_{1}+a_{2}+a_{3}\right)$. It is easy to see that the net increment vehicles for city 1,2 , and 3 are $-\left(a_{1}+a_{2}\right), a_{2}-a_{3}$ and $a_{1}+a_{3}$, respectively. Now we modify the transfer policy as:

If $a_{2}-a_{3} \geq 0$, transfer $a_{1}+a_{3}$ and $a_{2}-a_{3}$ cars from city 1 to city 2 and 3 , respectively to make sure there is no movement between city 2 and city 3 . It is readily to see that 
the net increment cost in these three cities are the same with the above transfer policy, but the total transfer cost is $k\left(a_{1}+a_{2}\right)$ which is less than $k\left(a_{1}+a_{2}+a_{3}\right)$;

$$
\text { If } a_{2}-a_{3}<0 \text {, transfer } a_{1}+a_{2} \text { cars from city } 1 \text { to city } 2
$$

and $a_{2}-a_{3}$ cars from city 3 to city 2 , respectively to insure no movement occurs between city 1 and city 2 . One can easily find that the net increment vehicles in these three cities keep unchanged when comparing with the above policy, but the overall transfer cost is $k\left(a_{1}+a_{3}\right)$ which is also less than $k\left(a_{1}+a_{2}+a_{3}\right)$

Case 2): ${ }^{a_{1}}, a_{2}, a_{3}$ cars are transferred from city 1 to city 2 , from city 2 to city 3 and from city 3 to city 1, respectively. Therefore, the net increment vehicles for city 1, 2, and 3 are $a_{3}-a_{1}, a_{1}-a_{2}$, and $a_{2}-a_{3}$, respectively; and the total transfer cost is $k\left(a_{1}+a_{2}+a_{3}\right)$. Without loss of generality, assume $\max \left(a_{1}, a_{2}, a_{3}\right)=a_{1}$, we modify the transfer policy as:

If $a_{2}-a_{3} \geq 0$, transfer $a_{1}-a_{2}$ and $a_{2}-a_{3}$ cars from city 1 to city 2 and city 3 , respectively to insure there is no movement between city 2 and city 3 . It is readily seen that the net increment vehicles in these three cities are the same with the above transfer policy, but the total transfer cost is $k\left(a_{1}-a_{3}\right)$ which is less than $k\left(a_{1}+a_{2}+a_{3}\right)$;

$$
\text { If } a_{2}-a_{3}<0 \text {, transfer } a_{1}-a_{3} \text { cars from city } 1 \text { to city } 2
$$

and $a_{3}-a_{2}$ cars from city 3 to city 2 , respectively and there is no movement between city 1 and city 3. Again, one can easily find that the net increment vehicles in these three cities keep unchanged when comparing with the above policy, but the overall transfer cost is $k\left(a_{1}-a_{2}\right)$ which is less than $k\left(a_{1}+a_{2}+a_{3}\right)$.

Hence, we complete the proof.

We can see clearly that in Lemma 1 of Li and Tao (2010), when $a_{1}{ }^{*} a_{2}^{*} \neq 0$, it indicates that in a two-city model, transfer activity from city 2 to city 1 and from city 2 to city 1 coexist, which can be seen as they formulate a ring. Therefore, it is a special case of our conclusion. Based on Lemma 1, we can propose the following observation:

Proposition 1. For each city, it is in only one of the following state:

Replenish the vehicles from other city (cities);

Transfer vehicles to other city (cities);

\section{No inbound and outbound occurs.}

It is easy to prove the above proposition.

In multi-city transfer problem, company may have to transfer vehicles from some cities to others, which is different from two-city transfer model. In two-city transfer problem, when one city is lack of vehicles, it has to be replenished from the other one, also the only one choice. However in multi-city transfer case, there may not be necessary to take any transfer activity in some city (or cities) while the vehicles may need to be replenished in some city (cities) or need to be slashed down in other city (cities). Therefore, the extra inventory of one city may not equal to the stock-out of another one city, which indicates that we may have to transfer vehicles from one city to two or more cities or from two or more cities to one city. Hence, it is better to design a transfer policy to minimize the total allocation cost. Based on the above conclusions, given the number of vehicles to be moved in each city, we propose a transfer algorithm as follows:

\section{Algorithm:}

If all cities have been involved, stop; otherwise choose any three uninvolved cities, draw a figure to reflect the transfer activities according to Proposition 1;

If these three cities achieve a balance (it indicates that no more vehicles are needed in these three cities and no more vehicles in these three cities have to be moved to other ones), go to step 1; otherwise go to next step;

Add a new point which has not involved;

Adjust the transfer policy according to Proposition 1, if all points contained so far can keep balance, go to step 1, otherwise go to step 3 .

\section{CONCLUSION}

This paper extends the two-city rental car problem into multi-city network, which also consists of inter-city and localcity demand. A dynamic programming model is proposed. The principle of the transfer activity is analysed. As the complexity of multi-city network, it is intractable to identify the analytical solution of this problem such that simulation and computational study of this problem is worthy of further investigation.

\section{ACKNOWLEDGEMENT}

This research is supported by National Science Foundation of China (Project No. 71201059), Postdoctoral Foundation of China (Project No.2011M500745) and the Fundamental Research Funds for the Central Universities.

\section{REFERENCES}

[1] Li, Z. \& Tao, F., On determining optimal fleet size and vehicle transfer policy for a car rental company. Computers and Operations Research, 37, pp. 341-350, 2010.

[2] Oliveria, B.B., Carravilla, M.A., Oliveira, J.F. \& Toledo, F.M.B., A relax-and-fix-based algorithm for the vehicle-reservation assignment problem in a car rental company. European Journal of Operational Research, 237, pp. 729-737, 2014. 
[3] You, P.S. \& Hsieh, Y.C., A study on the vehicle size and transfer policy for car rental problems. Transportation Research Part E, 64, pp. 110-121, 2014.

[4] Lin, J.T., Wu, C.H. \& Huang, C.W., Dynamic vehicle allocation control of automated material handling system in semiconductor manufacturing. Computers and Operations Research, 40, pp. 2329-2339, 2013.

[5] Goldbarg, M.C., Asconavleta, P.H. \& Goldbarg, E.F.G., Memetic algorithm for the traveling car renter problem: an experimental investigation. Memetic Computing, 4, pp. 89-108, 2012.

[6] Awasthi, A., Chauhan, S.S., Parent, M. \& Proth, J.M., Centralized fleet management system for cybernetic transportation. Expert Systems with Applications, 38, pp. 3710-3717, 2011. 